\title{
Videobronchoscope-assisted repair of the membranous tracheal laceration during insertion of a tracheostomy tube after tracheostomy
}

\author{
Shinichiro Okada, MD, Shotaro Ishimori, MD, \\ Shunsuke Yamagata, MD, Shoichi Satoh, MD, \\ Yoshiaki Tanaba, MD, and Sadanori Yaegashi, MD, \\ Kamaishi, Japan
}

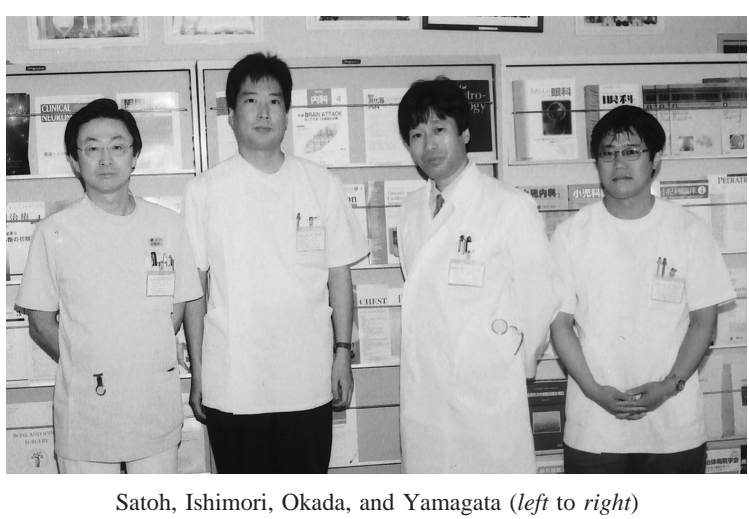

The patient was placed in the supine position, with her neck hyperextended. Oxygen was given with a face mask. Diazepam, 5 $\mathrm{mg}$, was given intravenously. The tracheostomy tube was removed, and the fifth and sixth tracheal rings of the anterior tracheal wall were incised longitudinally in the midline for further exposure. A fiberoptic flexible bronchoscope (BF P40; Olympus, Tokyo, Japan) was inserted through the incision. The edges of the incised anterior tracheal wall were retracted laterally. With the assistance of fiberoptic videobronchoscopic vision, a needle holder was introduced into the trachea and the target lesion. The lacerated lesion was repaired with running polydioxanone monofilament sutures (4-0 PDS II; Ethicon, Inc, Tokyo, Japan) during videobronchoscopic vision. The incised tracheal rings were sutured with interrupted polydioxanone sutures (3-0 PDS II, Ethicon). The tracheostomy tube was cautiously replaced into the trachea. Blood pressure, percutaneous oxygen saturation, and electrocardiography were not changed during the procedure. The operative procedure was uneventful. The operating time was 50 minutes. Bronchoscopy 2 weeks after the operation showed no signs of infection or anastomotic breakdown. An examination with a fiberoptic flexible bronchoscope performed 1 month after the operation showed an almost unnoticeable tracheal suture line. The cosmetic result was excellent.

\section{Discussion}

Iatrogenic lacerations of the trachea are uncommon. These injuries occur as a complication of endotracheal intubation, tracheostomy, ${ }^{1-3}$ median sternotomy, ${ }^{4}$ endotracheal stenting (eg, a Dumon stent), ${ }^{5}$ or operative procedures (eg, transhiatal blunt esophagectomy). In our case the fact that symptoms, such as stridor and dyspnea, occurred during the placement of a tracheostomy tube and tracheostomy suggests that the laceration of the trachea occurred as a result of the tracheostomy or traumatic insertion of the tracheostomy tube combined with weakness of the tracheal wall.

Early recognition and emergency repair are essential because failure to do so could result in potentially lethal events. In case of suspected tracheal lacerations, flexible bronchoscopy should be performed as soon as possible to confirm the diagnosis and to determine the exact location and extent of the lesion. Although treatment can be conservative for small lacerations of the membranous trachea or when less than one third of the circumference of the trachea or bronchus is disrupted, surgical repair is indicated in case of extensive lacerations or disruption of more than one third of the circumference. ${ }^{2}$ Although long-term prognosis de- 
pends more on the underlying disease than on the injury itself, immediate postoperative results usually are satisfactory. Therefore, urgent surgical repair of tracheal tears should be the rule. The surgical approach is determined on the basis of the location of the tracheal lesion. ${ }^{1,2}$ Left cervicotomy is performed for the cervical trachea, with partial division of the sternum, if necessary, for further exposure, and right thoracotomy in the fourth intercostal space for the thoracic part. Lesions of the lower half of the trachea, particularly of the membranous posterior wall, are most easily approached through a high right thoracotomy incision. Additional cover might be required when tissues are inflammatory or when early extubation is impossible. Various flaps, such as intercostal muscle, pericardial graft, and a fatty pad, have been used to reinforce primary closures and to bridge small defects. ${ }^{2}$ In our case the transcervical and transtracheal approach with flexible videobronchoscopic guidance provided a good exposure and allowed for a precise suturing of the laceration. The technique required no thoracotomy incision. Additionally, although the procedure was performed under emergency conditions, it was easy to perform.
Careful manipulation to avoid injuring the trachea during insertion of a tracheostomy tube after a tracheostomy is mandatory during the procedure. Our procedure with the aid of a fiberoptic flexible bronchoscope might be feasible in selected patients with a tracheal membranous laceration.

\section{References}

1. Massard G, Rouge C, Dabbagh A, Kessler R, Hentz JG, Roeslin N, et al. Tracheobronchial lacerations after intubation and trachesostomy. Ann Thorac Surg. 1996;61:1483-7.

2. Marty-Ane $\mathrm{CH}$, Picard E, Jonquet O, Mary H. Membranous tracheal rupture after endotracheal intubation. Ann Thorac Surg. 1995;60:1367-71.

3. Angelillo-Mackinlay T. Transcervical repair of distal membranous tracheal laceration. Ann Thorac Surg. 1995;59:531-2.

4. Takanami I. Tracheal laceration: a rare complication of median sternotomy. J Thorac Cardiovasc Surg. 2001;122:184.

5. Niwa H, Masaoka A, Yamakawa Y, Fukai I, Kiriyama M, Shindou J. Esophageal tracheobronchoplasty for membranous laceration caused by insertion of a Dumon stent. Maintenance of oxygenation by percutaneous cardiopulmonary support. Eur J Cardiothoracic Surg. 1995;9:213-5. 